\title{
Epidemiology of adolescent total knee arthroplasty patient and a comparison to adult patient demographics
}

\author{
Samantha Nguyen ${ }^{1}$, Katelyn Pratt ${ }^{1}$, Mouhanad M El-Othmani*2 ${ }^{* 2}$ Tony Tzeng ${ }^{2}$, Steven Scaife ${ }^{3}$, Sonia Vasdev ${ }^{2}$, Khaled \\ Jamal Saleh ${ }^{2}$ \\ ${ }^{1}$ Southern Illinois University School of Medicine, Springfield, United States \\ ${ }^{2}$ Division of Orthopaedics and Rehabilitation, Department of Surgery, Southern Illinois University School of Medicine, \\ Springfield, United States \\ ${ }^{3}$ Center for Clinical Research, Southern Illinois University School of Medicine, Springfield, United States
}

Received: July 15, 2015

DOI: $10.5430 /$ css.v2n1p1
Accepted: August 5, 2015

URL: http://dx.doi.org/10.5430/css.v2n1p1
Online Published: September 2, 2015

\begin{abstract}
Background/Objective: An increasing prevalence of total knee arthroplasty (TKA) procedures among the adolescent population makes it critical to establish a greater understanding of the patient and hospital demographics of this population. The purpose of this study is to compare inpatient demographics between the adolescent and adult populations as well as assess patient and hospital demographics at which adolescent TKA procedures are performed.

Methods: Kids' Inpatient Database (KID) and Nationwide Inpatient Sample (NIS) database were used to retrieve data for all TKA patients under 20 years and over the age of 65, respectively. Variables analyzed included patient age, gender, ethnicity, primary diagnosis, length of stay, primary payer status, total hospital charges, complication rates, and hospital status.

Results: The study found a significant increase in procedure frequency (156 to 448), complication rates (3.51\% to $17.61 \%)$, and total charges $(\$ 38,776$ to $\$ 128,811)$ for adolescent TKA patients. Some trends in demographics and outcomes for adolescent TKAs differed from their adult counterparts, as the primary diagnosis, hospital status, gender, and payer status. Additionally, the number of procedures, complication rates, length of stay, and charges were all significantly higher in adolescent than in adult TKAs.

Conclusions: Although the TKA procedure rates are rising in the adolescent population, the lack of available demographic information limits the efficacy of the procedure in the younger population, presenting a challenge for health-care providers. Further studies should focus on whether epidemiological disparities, which have been noted in adults, also exist in the adolescent patient population.
\end{abstract}

Key Words: Total knee arthroplasty, Epidemiology, Demographics, Pediatric, Adolescent

\section{INTRODUCTION}

Total knee arthroplasty (TKA) is a common procedure used to treat disorders that impair the joint's mobility including arthritis, joint deformity, and traumatic knee injury. ${ }^{[1-5]} \mathrm{Al}-$ though TKA is typically performed on adult patients, it is also utilized to treat pediatric diseases affecting the knee joint

\footnotetext{
*Correspondence: Mouhanad M El-Othmani; Email: mohannad.othmani@gmail.com; Address: Division of Orthopedics and Rehabilitation, Southern Illinois University School of Medicine, P.O. Box 19679, Springfield, United States.
} 
such as childhood osteosarcoma, advanced juvenile rheumatoid arthritis (JRA), and osteoarthritis. ${ }^{[6-12]}$ Despite high rates of complications and increased likelihood of revision in the younger population, the procedure has high success rates in providing pain relief, increasing joint function and improving quality of life for those who suffer from degenerative knee disorders. ${ }^{[9,13-16]}$

The incidence of primary TKA in the US population has increased between 1990 and 2002 from 129,000 to 381,000. ${ }^{[14]}$ TKA procedures in the United States are estimated to rise by $673 \%$ or more by $2030 .{ }^{[14,17]}$ In the past, there have been numerous studies on the epidemiology and efficacy of TKA in the elderly population (patients over 65 years); however, few studies looked at the epidemiology and use of TKA in the young adult or pediatric populations. ${ }^{[5,9,11,15,18,19]}$

Although TKA procedure frequency has been increasing in the adolescent population, information regarding this population's primary indications and demographics is still limited. ${ }^{[9]}$ The purpose of this study is to explore trends in the patient and hospital demographics of adolescent patients undergoing TKA procedures and to compare them to inpatient characteristics of elderly TKA patients. These findings will be important in delineating patient and hospital demographic factors and trends in procedure utilization rates.

\section{MeTHODS}

Institutional Review Board exemption was obtained for this study from the Springfield Committee for Research Involving Human Subjects (SCHRIS). Weighted data was obtained from the Kids' Inpatient Database (KID) and the Nationwide Inpatient Sample (NIS). The KID is the only all-payer national inpatient database for children 20 years of age and younger, and the NIS is the largest all-payer national inpatient database that contains information from over eight million patients of 46 states. KID data, which has been available every three years since 1997, is extracted through discharge information from 2,500 to 4,000 hospitals and covers 2 to 3 million patients. Data used for the study was obtained from the years 1997, 2000, 2003, 2006, and 2009 from KID and the year 2009 from NIS. The comparison between the KID and NIS was performed using the 2009 databases. The population examined in this study consisted of patients younger than 20 years of age (KID) and over the age of 65 (NIS) who underwent TKA.

Using the International Classification of Diseases-9 (ICD 9) code for TKA (81.54) information such as patient age, gender, ethnicity, primary diagnosis, length of stay, primary payer status, hospital discharge, and hospital total charges was extracted (see Table 1).
The data was analyzed using SAS 9.2 with the FREQ procedure. The FREQ procedure produces frequency and cross tabulation tables and computes statistics for contingency tables which include chi-square tests. $P$ values less than .05 were considered to be statistically significant. Results were weighted to obtain accurate comparison of statistics at different time points.

Table 1. Variables extracted from KID and NIS databases

\begin{tabular}{ll}
\hline Independent Variables & \\
\hline Age & Total Charges \\
$<20$ & \\
$>65$ & Complication Rate
\end{tabular}

\begin{tabular}{|c|c|}
\hline Gender & Length of Stay \\
\hline \multicolumn{2}{|l|}{ Male } \\
\hline Female & Primary Diagnosis $^{\#}$ \\
\hline Race $^{*}$ & Hospital Teaching Status \\
\hline Caucasian/Non-Caucasian & Teaching \\
\hline African American & Non-teaching \\
\hline \multicolumn{2}{|l|}{ Hispanic } \\
\hline Asian/Pacific Islander & Disposition \\
\hline Native American & Routine \\
\hline Other & $\begin{array}{l}\text { Transfer to short-term hospital } \\
\text { Other Transfer }\end{array}$ \\
\hline Primary Payer Type & Home Health Care \\
\hline \multicolumn{2}{|l|}{ Medicare } \\
\hline \multicolumn{2}{|l|}{ Medicaid } \\
\hline \multicolumn{2}{|l|}{ Private } \\
\hline \multicolumn{2}{|l|}{ Self-Pay } \\
\hline Other & \\
\hline
\end{tabular}

Note. ${ }^{*}$ In adolescents, ethnicity was reported as Caucasian and non-Caucasian groups due to small patient populations limiting reportable data. The non-Caucasian group included African Americans, Hispanics, Asian/Pacific Islanders, Native Americans, and other. ${ }^{\#}$ In adolescents, primary diagnosis was classified according to whether it was a malignant neoplasm or other due to small patient populations limiting reportable data. Other diagnoses included secondary or unknown neoplasm, osteoarthritis, juvenile chronic polyarthritis, rheumatoid arthritis, mechanical complication of an internal device/implant, and aseptic necrosis of the bone.

\section{RESULTS}

The number of TKA procedures reported in the KID database increased from 156 cases in 1997 to 448 procedures in 2009 (see Figure 1). In this population, TKA procedures were most often performed in the age-group 12 and 20 years. The top diagnosis throughout this period was malignant neoplasm of long bones of the lower limb, and the procedure was most often performed at teaching hospitals. Complication rates significantly increased from $3.51 \%$ in the 1997 KID to $17.61 \%$ 
in the 2009 KID ( $p=.0007$; see Figure 1). Length of stay in the hospital following the procedure decreased overall between 1997 and 2009, however this difference was not statistically significant (see Figure 2).

In our population, $61.5 \%$ of the patients reported their ethnicity as Caucasian (see Figure 3). In 1997, 45\% of KID TKA patients were male while $55 \%$ were female. The number of male patients increased to greater than $50 \%$ for all other years included in this study (see Figure 4). In 1997, primary payer status was dominated by patients in the private (including HMO) category at $74.56 \%$ (see Figure 5). This percentage significantly decreased to $55.68 \%$ of KID TKA patients by 2009 ( $p<.0001)$. Total charge increased significantly between the 1997 and 2009, from $\$ 38,776$ to $\$ 128,811$ ( $p<.0001)$, respectively. Procedure charges also increased significantly between 2003 and 2006 and again between 2006 and $2009(p<.05)$ (see Figure 6).

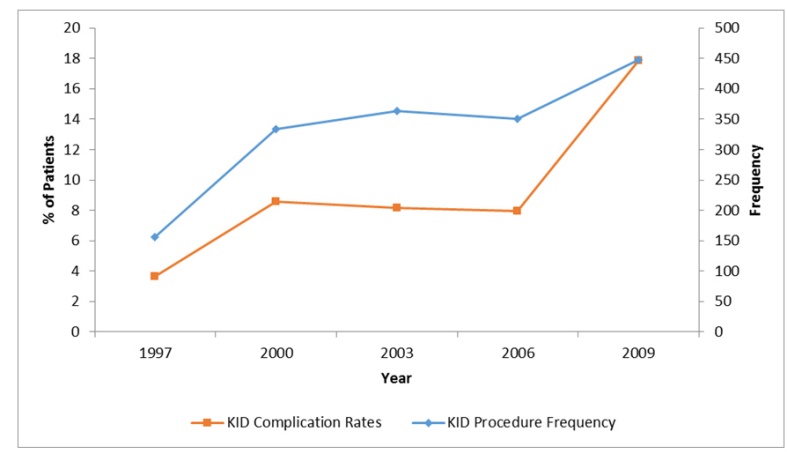

Figure 1. TKA procedures in KID



Figure 2. Mean pediatric length of hospital stay

Data comparison of TKA procedures between the 2009 KID and NIS databases revealed significant demographic shifts (see Table 2). More procedures were performed in the adult population than the pediatric population (see Table 2). The top primary diagnosis for the procedures in the 2009 KID was malignant neoplasm of long bones of the lower limb while osteoarthritis was the top diagnosis for TKA in the 2009 NIS. A majority of KID TKA procedures were performed in teaching hospitals during 2009, while the majority of NIS TKA procedures were performed in non-teaching hospitals (see Table 2). Complication rates were significantly higher in the adolescent population than adult patients in 2009 $(p<.0001)$. Length of stay was also significantly higher in the pediatric group, with adolescent patients remaining hospitalized nearly twice as long as adult patients $(p<.0001)$. In terms of disposition, a significantly higher number of TKA patients in the KID sample received routine discharges than those in the NIS group $(p<.0001)$.

Table 2. KID vs. NIS demographics

\begin{tabular}{lll}
\hline & 2009 KID & 2009 NIS \\
\hline Frequency & 448 & 354,952 \\
Top Primary Diagnosis & Malignant & Osteoarthritis \\
& Neoplasm & \\
\% Caucasian Patients & $62.83 \%$ & $85.43 \%$ \\
\% Male Patients & $52.29 \%$ & $36.35 \%$ \\
Total Charges & $\$ 128,811$ & $\$ 46,180$ \\
Complication Rate & $17.84 \%$ & $6.39 \%$ \\
Mean Length of Stay (days) & 6.41 & 3.46 \\
Primary Payer Status & Private & Medicare \\
Primary Hospital Teaching & including HMO & \\
Status & Teaching & Non-teaching \\
\% Routine Dispositions & $64.20 \%$ & $19.56 \%$ \\
\hline
\end{tabular}

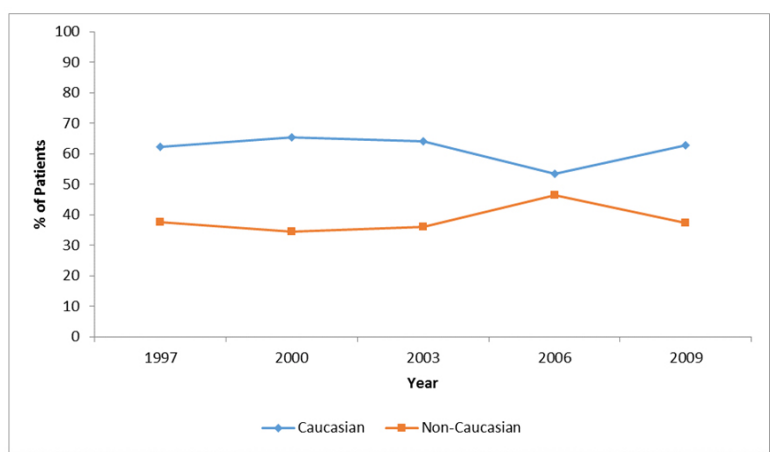

Figure 3. Pediatric ethnicity trends in TKA. The blue line with stars represents Caucasian patients while the red line with squares shows the Non-Caucasian group.

As shown in Table 2, a significantly higher proportion of Caucasian patients was reported in the adult population compared to those in the KID sample $(p<.0001)$. The majority of adult patients undergoing TKA were female, while most adolescent patients undergoing the same procedure were male $(p<.0001)$. A significantly higher number of private payer was reported in the KID database when compared to the NIS sample $(p<.0001)$. In addition, the total charge for 
procedures performed in adolescents was nearly three times that of the adults $(p<.0001)$.

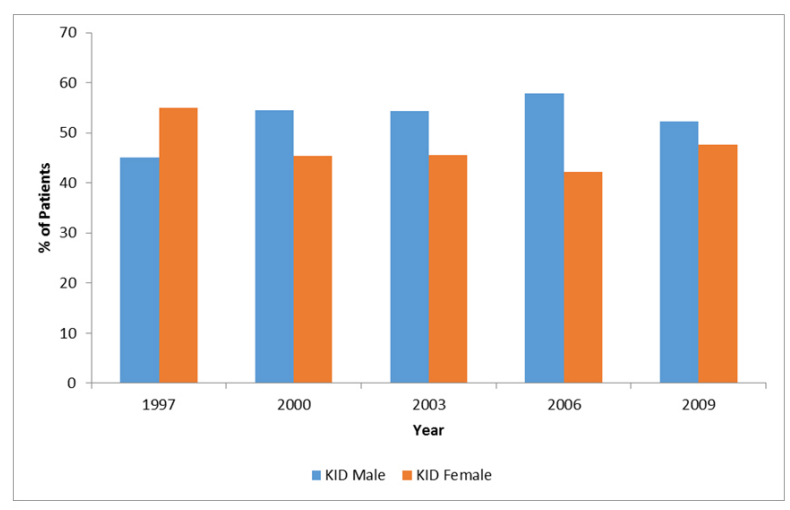

Figure 4. Pediatric gender trends in TKA. In blue are males and red represents females.

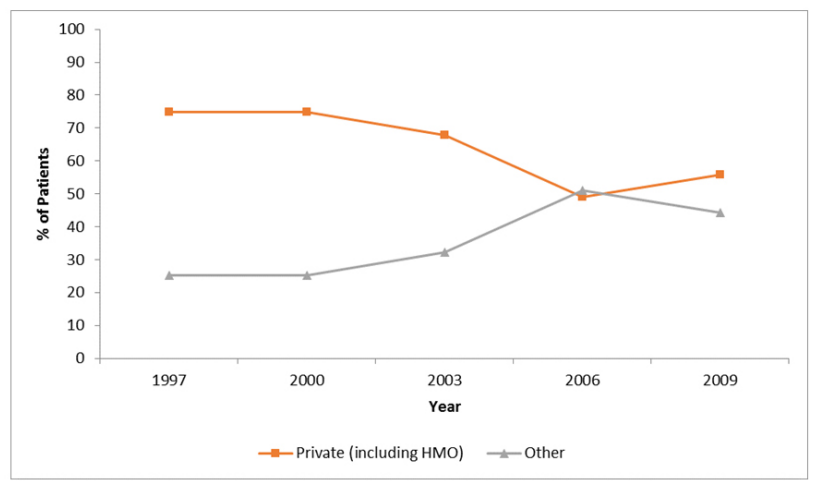

Figure 5. Primary Payer Status in Pediatric TKA. The Private group is represented in red and squares, while the green line and triangles represent the Other category.

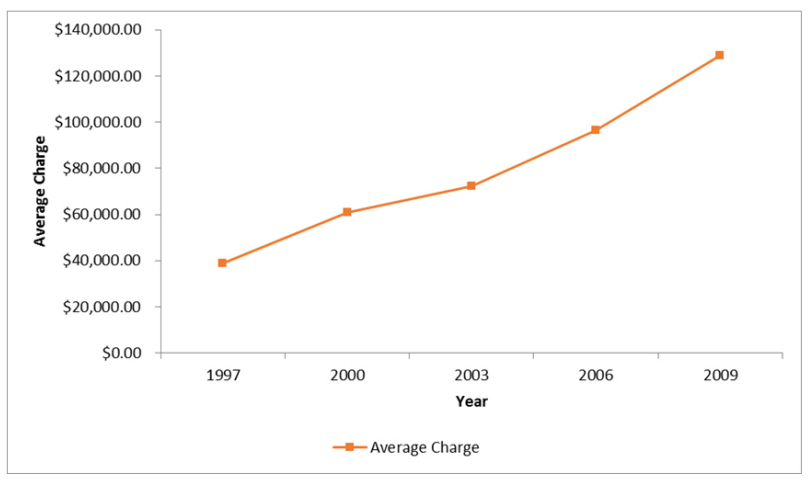

Figure 6. Mean pediatric total hospital charges

\section{Discussion}

Very few studies have reported the demographic distribution of the adolescent TKA population. To the authors' knowledge, this is the first study examining patient and hospital demographics of adolescent patients undergoing total knee replacements and comparing them to similar demographics in the adult population.

Osteosarcoma is an extremely aggressive type of malignant bone tumor that is most often seen in the metaphysis of long bones of the lower limbs. ${ }^{[10,20,21]}$ About half of the cases involve the knee as the site of disease, among which $40 \%$ involve the metaphysis of the distal femur and $21 \%$ are located in the proximal tibia. ${ }^{[1,7]}$ Osteosarcoma has a bimodal age distribution, with $40 \%-60 \%$ of cases diagnosed around the adolescent growth spurt phase, at approximately the age of 15 years for females and 18 years for males. ${ }^{[10,21,22]}$ Though the peak age of diagnosis is younger in females than males, the prevalence of osteosarcoma is actually 1.4 times higher in males, with rates being higher in African-American children compared to Caucasians. ${ }^{[10,20-22]}$

Because malignant neoplasms are currently the top primary diagnosis indicating TKA procedure, demographics of osteosarcoma may help explain some of the trends reported in the KID database. However, since the database does not allow the analysis of prior admissions, it is difficult to elucidate the decision-making behind these procedures. Since the year 2000, the majority of patients undergoing TKA in the KID have been males, which is consistent with the gender distribution of osteosarcoma. ${ }^{[10,20-22]}$ The higher incidence of disease during the growth spurt age-range led some studies to indicate a correlation between rapid bone growth and the development of the disease. ${ }^{[10]}$ In addition, the majority of adolescents undergoing the procedure are between the ages of 12 and 20 years, which is centralized around the adolescent growth spurt age-range. ${ }^{[10,20,21]}$ No correlation could be found between primary payer status and mean total charges, suggesting that increasing costs are not due to a lack of coverage by private insurances. Therefore, another factor outside of those previously hypothesized is influencing the change in primary status and mean total charges. Factors such as the type of implant-components used in adolescent TKA procedures, in relation with increased total charges could be examined in future studies. Since the first procedure in the late 1970s, total knee replacements have undergone technological improvements and become the standard of care for osteosarcomas. ${ }^{[10]}$ New developments, such as expandable prostheses, are also allowing TKA, which is typically not considered as an option until teenage years due to skeletal immaturity, to be performed in even younger osteosarcoma patients. ${ }^{[10]}$ Due to these technological advances and the high cost associated with these implants, the cost of knee replacements have increased by more than $100 \%$ in the last decade. ${ }^{[23,24]}$ Though the KID database reports total charges, it does not report on the type of prostheses used in total knee 
replacements, making it difficult to determine a correlation between cost and implant-type.

High rates of complications remain a significant cause of morbidity and mortality, as seen in the KID dataset, which showed an increase in complication rates from $3.51 \%$ in 1997 to $17.61 \%$ in $2009(p<.0007){ }^{[10,12]}$ One factor that could be contributing to this increase is the technology-advances which has allowed total knee replacements to be performed in younger children. ${ }^{[10]}$ Skeletal immaturity in younger patients increases rates of complications, impairing their future growth and treatment. ${ }^{[7,10]}$ Another factor that could be influencing complication rates is the teaching status of the hospital where TKA is being performed and the complexity of the procedure. Since 2000 , over $80 \%$ of TKA procedures in the KID have been performed in teaching hospitals and approximately $93 \%$ of complications have occurred in those same locations $(p<.02)$.

Although an increase has been observed in complication rates and mean total charges, length of stay and routine disposition have not followed that same trend. A significant correlation was found between total charges and length of stay $(p<.0001)$, but not between total charges and complication rates $(p=.7384)$. Future research is needed to determine how routine disposition and length of stay have remained consistent, despite significant and independent increases in total charges and complication rates.

Our study found that, consistent with other studies, the top diagnosis for patients over 65 receiving TKA was osteoarthritis. ${ }^{[25-27]}$ Osteoarthritis prevalence is significantly higher and often more debilitating in women than men, thus resulting in higher incidence of TKA procedures among women. ${ }^{[28]}$ This is confirmed with our findings of only $36 \%$ of adult TKA patients being male in 2009 .

Table 2 displays the significant differences in demographic trends between adult and adolescent patients undergoing TKA. We hypothesized that the differences in gender and ethnicity between the two groups were related to the characteristics of each group's top diagnosis. Osteosarcoma is typically found in adolescent males, while osteoarthritis is primarily seen in adult females. ${ }^{[28,29]}$ This is consistent with the $62 \%$ of adolescent males versus $36 \%$ of adult male patients undergoing TKA in our KID and NIS database reviews, respectively. ${ }^{[10,20-22]}$ Though both diseases have a higher incidence in ethnicities other than Caucasian, in this study the majority of TKA patients in both groups are Caucasian. Further review is needed to determine why both age groups of patients are so predominantly Caucasian despite disease predilections. ${ }^{[30-32]}$
Significant differences between the adolescent and adult patients in categories such as primary payer, length of stay, and disposition type, are hypothesized to be influenced by patient age. In 2009 , nearly $56 \%$ of KID patients reported private payer status while only $11 \%$ of NIS patients reported private insurance as their primary payer. Matlock et al. found that prior to age 65 , most TKA procedures were paid by private insurance carriers, while more than $80 \%$ of procedures for patients older than 65 were paid for by Medicare. ${ }^{[33]}$ This is consistent with our finding of a significantly lower percentage of private payer status in the NIS than in the KID datasets $(p<.0001)$. There is also a noticeable increase in procedure utilization at age 65, most likely a result of Medicare benefits going into effect for all patients over 65 , including those who were previously uninsured. ${ }^{[33,34]}$

The analysis of the NIS database showed a correlation between private primary payer status and both length of stay and total charge $(p<.0001)$. This relation was not seen in the KID analysis, where total charge only was correlated with length of stay in adolescents. The difference between the two populations could be due to the significant difference in private insurance utilization; however, private payer primary status was not found to be correlated with either length of stay or total charge in adolescents. The length of stay for adult populations $(p<.0001)$ was almost half of the average length of stay for adolescents. This may be explained by looking at the difference in disposition type between the two populations: $64.2 \%$ of KID patients compared to the $19.6 \%$ of NIS patients received routine dispositions $(p<.0001)$. One study found that many adult patients were being discharged to other rehabilitation or short-term stay facilities, thus reducing the amount of time spent in the hospital following TKA, and decreasing total charges incurred while in-hospital. ${ }^{[35]}$ Though length of stay would contribute to the significantly lower total charge for adult patients compared to adolescents $(p<.0001)$, an additional effect can be caused by the type of prosthesis used. The newer implants needed in younger patients might be more expensive than older or less complex models used in the adults.

This study was limited by the reporting capacity of the KID and NIS databases. Though these datasets have grown to include a majority of hospitals, not all 50 states are included in these databases. Additionally, hospitals reporting data may not consistently provide information for every variable that is listed in each respective database. There is also a paucity of literature discussing epidemiology of total knee replacements in adolescents and none comparing the demographics between the adolescent patient population and typical adult patient population. This makes it more difficult to confirm that correlations and statistical significance have been estab- 
lished elsewhere.

With utilization rates of TKA increasing dramatically, an understanding of which population subgroups are at the highest risk of requiring TKA is needed. TKA utilization in pediatric patients is less than in adults, but the lack of available demographic information presents a challenge for healthcare providers wanting to know about procedure efficacy in the younger population. Epidemiological disparities have been noted within the adults, and it is important to determine whether they also exist in the adolescent patient population. Further studies should focus on how demographics between the two populations may differ as well as the changing epidemiology of adolescent patients undergoing total knee replacements.

\section{REFERENCES}

[1] Crowder AR, Duffy GP, Trousdale RT. Long-term results of total knee arthroplasty in young patients with rheumatoid arthritis. The Journal of arthroplasty. 2005; 20 (7 Suppl 3): 12-16. http: //dx.doi.org/10.1016/j.arth.2005.05.020

[2] Dalury DF, Ewald FC, Christie MJ, et al. Total knee arthroplasty in a group of patients less than 45 years of age. The Journal of arthroplasty. 1995; 10(5): 598-602. http://dx.doi .org/10.1016/S08 83-5403(05) 80202-5

[3] Diduch DR, Insall JN, Scott WN, et al. Total knee replacement in young, active patients. Long-term follow-up and functional outcome. The Journal of bone and joint surgery American. 1997; 79(4): 575582.

[4] Gioe TJ, Novak C, Sinner P, et al. Knee arthroplasty in the young patient: survival in a community registry. Clinical orthopaedics and related research. 2007; 464: 83-87. http://dx.doi.org/10.10 97/BLO. 0b013e31812f79a9

[5] Stuart MJ, Rand JA. Total knee arthroplasty in young adults who have rheumatoid arthritis. The Journal of bone and joint surgery American. 1988; 70(1): 84-87.

[6] Futani H, Minamizaki T, Nishimoto Y, et al. Long-term followup after limb salvage in skeletally immature children with a primary malignant tumor of the distal end of the femur. Journal of Bone and Joint Surgery-American. 2006; 88(3): 595-603. http: //dx.doi.org/10.2106/JBJS.C. 01686

[7] Grimer RJ. Surgical options for children with osteosarcoma. The lancet oncology. 2005; 6(2): 85-92. http://dx.doi.org/10.10 16/S1470-2045(05) 01734-1

[8] Keeney JA, Eunice S, Pashos G, et al. What is the evidence for total knee arthroplasty in young patients?: a systematic review of the literature. Clinical orthopaedics and related research. 2011; 469(2): 574-583. http://dx.doi.org/10.1007/s11999-010-1536-9

[9] Li XWA, Iyer S, Cross MB, et al. Total joint replacement in adolescents: literature review and case examples. Current opinion in pediatrics. 2012; 24(1): 57-63. http://dx.doi.org/10.1097/M OP. 0b013e32834ec96a

[10] Marina N, Gebhardt M, Teot L, et al. Biology and therapeutic advances for pediatric osteosarcoma. The oncologist. 2004; 9(4): 422441. PMid: 15266096 . http://dx.doi.org/10.1634/theonco logist.9-4-422

[11] Palmer DH, Mulhall KJ, Thompson CA, et al. Total knee arthroplasty in juvenile rheumatoid arthritis. The Journal of bone and joint surgery American. 2005; 87(7): 1510-1514. http://dx.doi.org/10.21 06/JBJS.D. 02200

[12] Rojer DE, Goodman SB. Total knee replacement in juvenile rheumatoid arthritis. Orthopedics. 2005; 28(1): 39-45; quiz 46-37.

[13] Jolles BM, Bogoch ER. Quality of life after TKA for patients with juvenile rheumatoid arthritis. Clinical orthopaedics and related re- search. 2008; 466(1): 167-178. http://dx.doi.org/10.1007/s 11999-007-0010-9

[14] Kurtz S, Mowat F, Ong K, et al. Prevalence of primary and revision total hip and knee arthroplasty in the United States from 1990 through 2002. The Journal of bone and joint surgery American. 2005; 87(7): 1487-1497. http://dx.doi.org/10.2106/JBJS.D. 02441

[15] Parvizi J, Lajam CM, Trousdale RT, et al. Total knee arthroplasty in young patients with juvenile rheumatoid arthritis. The Journal of bone and joint surgery American. 2003; 85-A(6): 1090-1094.

[16] Hawker GA. Who, when, and why total joint replacement surgery? The patient's perspective. Current opinion in rheumatology. 2006; 18(5): 526-530. http://dx.doi.org/10.1097/01. bor.000024 0367.62583 .51

[17] Kurtz S, Ong K, Lau E, et al. Projections of primary and revision hip and knee arthroplasty in the United States from 2005 to 2030. The Journal of bone and joint surgery American. 2007; 89(4): 780-785. http://dx.doi.org/10.2106/JBJS.F.00222

[18] Mulhall KJ, Saleh KJ, Thompson CA, et al. Results of bilateral combined hip and knee arthroplasty in very young patients with juvenile rheumatoid arthritis. Archives of orthopaedic and trauma surgery. 2008; 128(3): 249-254. http://dx.doi.org/10.1007/s 00402-007-0450-4

[19] Sarokhan AJ, Scott RD, Thomas WH, et al. Total knee arthroplasty in juvenile rheumatoid arthritis. The Journal of bone and joint surgery American. 1983; 65(8): 1071-1080.

[20] Larsson SE, Lorentzon R. The incidence of malignant primary bone tumours in relation to age, sex and site. A study of osteogenic sarcoma, chondrosarcoma and Ewing's sarcoma diagnosed in Sweden from 1958 to 1968 . The Journal of bone and joint surgery British. 1974; 56B(3): 534-540.

[21] Mirabello L, Troisi RJ, Savage SA. International osteosarcoma incidence patterns in children and adolescents, middle ages and elderly persons. International journal of cancer Journal international du cancer. 2009; 125(1): 229-234. http://dx.doi.org/10.1002/ijc .24320

[22] Damron TA, Ward WG, Stewart A. Osteosarcoma, chondrosarcoma, and Ewing's sarcoma. Clinical orthopaedics and related research. 2007; 459: 40-47. http://dx.doi.org/10.1097/BLO.0b013e3 $18059 \mathrm{~b} 8 \mathrm{c} 9$

[23] Ilalov K, Cohn RM, Slover J. High-performance total knee replacement. Bulletin of the Hospital for Joint Disease. 2013; 71(1): 79-88.

[24] Robinson JC, Pozen A, Tseng S, et al. Variability in costs associated with total hip and knee replacement implants. The Journal of bone and joint surgery American. 2012; 94(18): 1693-1698. http://dx.doi.org/10.2106/JBJS.K.00355

[25] Singh JA. Epidemiology of knee and hip arthroplasty: a systematic review. The open orthopaedics journal. 2011; 5: 80-85. http: //dx.doi.org/10.2174/1874325001105010080 
[26] Singh JA, Vessely MB, Harmsen WS, et al. A population-based study of trends in the use of total hip and total knee arthroplasty, 19692008. Mayo Clinic proceedings Mayo Clinic. 2010; 85(10): 898-904. http://dx.doi.org/10.4065/mcp. 2010.0115

[27] Mehrotra C, Remington PL, Naimi TS, et al. Trends in total knee replacement surgeries and implications for public health, 1990-2000. Public Health Rep. 2005; 120(3): 278-282. PMid: 16134568.

[28] Bang H, Chiu YL, Memtsoudis SG, et al. Total hip and total knee arthroplasties: trends and disparities revisited. Am J Orthop (Belle Mead NJ). 2010; 39(9): E95-102. PMid: 21290031.

[29] Hawker GA, Wright JG, Coyte PC, et al. Differences between men and women in the rate of use of hip and knee arthroplasty. The New England journal of medicine. 2000; 342(14): 1016-1022. http://dx.doi.org/10.1056/NEJM200004063421405

[30] Katz JN. Patient preferences and health disparities. JAMA : the journal of the American Medical Association. 2001; 286(12): 1506-1509. PMid: 11572745. http://dx.doi.org/10.1001/jama.286.12 .1506
[31] Suarez-Almazor ME, Souchek J, Kelly PA, et al. Ethnic variation in knee replacement: patient preferences or uninformed disparity? Archives of internal medicine. 2005; 165(10): 1117-1124. http://dx.doi.org/10.1001/archinte.165.10.1117

[32] Lavernia CJ, Lee D, Sierra RJ, et al. Race, ethnicity, insurance coverage, and preoperative status of hip and knee surgical patients. The Journal of arthroplasty. 2004; 19(8): 978-985. PMid: 15586333. http://dx.doi.org/10.1016/j.arth.2004.03.028

[33] Matlock D, Earnest M, Epstein A. Utilization of elective hip and knee arthroplasty by age and payer. Clinical orthopaedics and related research. 2008; 466(4): 914-919. http://dx.doi.org/10.1007 /s11999-008-0122-x

[34] Kim S. Changes in surgical loads and economic burden of hip and knee replacements in the US: 1997-2004. Arthritis and rheumatism. 2008; 59(4): 481-488. http://dx.doi.org/10.1002/art. 235 25

[35] Forrest G, Fuchs M, Gutierrez A, et al. Factors affecting length of stay and need for rehabilitation after hip and knee arthroplasty. The Journal of arthroplasty. 1998; 13(2): 186-190. http://dx.doi.o rg/10.1016/S0883-5403 (98) 90097-3 